\title{
Correlation and the Density Matrix Approach to Inelastic Electron Holography in Solid State Plasmas
}

\author{
Peter Schattschneider* \\ Laboratoire MSS-Mat - CNRS-UMR8579, Ecole Centrale Paris, France. \\ Hannes Lichte \\ Institute of Structure Physics, \\ Triebenberg-Laboratory, Dresden University
}

\begin{abstract}
It has been argued that collective excitations in solid state plasmas are a good candidate to measure correlation lengths of electrons. The method of choice seems to be energy filtered electron holography in the transmission electron microscope (TEM). The interference fringes contain information on the partial coherence of those electrons and thus on their correlation length. Previous experiments showed surprisingly high coherence.

We calculate the density-density correlation function in the Al plasma excitation from the dynamic form factor and compare it to other estimates. For the Al plasma excitation, we find a small extension of $0.1-0.3 \mathrm{~nm}$ for the range over which the movement of charges is correlated, depending on the definition of that range.

Using the density matrix formalism, the coherence length of plasmon scattered fast electrons in the TEM is calculated and found to agree with experiment. It is shown that the small correlation length does not contradict the coherence length of $>10 \mathrm{~nm}$ found for electrons having excited a plasmon in Al. The difference of nearly 2 orders of magnitude can be traced back to the long range Coulomb interaction between probe and target electrons. Two unexpected predictions ensue from the present approach: Inelastic holography experiments should show strongly increased contrast of interfeence fringes in vacuo, i. e. outside the specimen, and contrast inversion of fringes in inelastic holograms of very small particles.
\end{abstract}

PACS numbers: 34.80.Pa, 71.45.Gm, 82.80.Pv

\section{INTRODUCTION}

The correlated movement of electrons in matter, as well as coherent superpositions of electron wave functions play a dominant role for the modelling of energy loss spectra (e. g. $[1,2]$ ) and in many quantum applications such as Josephson junctions, quantum tunneling, spintronics, nm-sized semiconductors, nanotubes and molecules, e. g. [3, 4]. A famous example is the electron correlation in metals. The accurate calculation of the correlation energy in the interacting electron gas is a notorious problem with many approximate solutions and unsatisfactory agreement with experiment. For a review, see e. g. [5] and references therein.

It is reasonable to assume that the movement of electrons is correlated over a distance of the order of magnitude of the Thomas-Fermi screening length. On the other hand, the plasmon as a collective excitation is often visualized as an in-phase movement of many electrons in the specimen, thus having infinite correlation length.

In this paper we set out to describe correlation in solid state plasmas and its relationship to inelastic electron

*On leave from Institute for Solid State Physics, TU Wien, A1040 Vienna, Austria; Electronic address: schattschneider@ifp. tuwien.ac.at scattering by use of the density matrix. As an example we take Al metal. We first discuss the different existing approaches to a definition of the spatial extension of a plasma excitation. Then we focus on the density-density correlation $p(\mathbf{r})$ i. e. the conditional probability to find an electron a distance $\mathbf{r}$ away from another one. We then show how $p(\mathbf{r})$ relates to the dynamic form factor $S(\vec{q}, E)$ which in turn is accessible by inelastic scattering experiments with fast electrons. Realizing that the inelastic scattering cross section for fast electrons is closely related to the mutual coherence of the scattered electrons we are then in a position to elaborate the relationship between $p(\mathbf{r})$ and the mutual object transparency. We can then re-analyse the inelastic interference experiments of Lichte and coworkers. We find that the large coherence length determined experimentally is precisely predicted by theory, and that this is consistent with the small correlation length in solid state plasmas. We predict some peculiar properties of the mutual coherence function and propose new holographic experiments as a test of the present predictions.

\section{THE DENSITY CORRELATION FUNCTION}

The density correlation $p(\mathbf{r})$ is defined in terms of the density operator $n(\mathbf{r})=\sum_{\mathbf{i}} \delta^{\mathbf{3}}\left(\hat{\mathbf{r}}_{\mathbf{i}}-\mathbf{r}\right)$ as the integral over 
the expectation value

$$
p(\mathbf{r})=\int d^{3} r^{\prime}\left\langle n\left(\mathbf{r}^{\prime}\right) n\left(\mathbf{r}+\mathbf{r}^{\prime}\right)\right\rangle
$$

where the sum is over all particles.

This definition can be extended to time-dependent correlations as

$$
p(\mathbf{r}, t)=\int d^{3} r^{\prime}\left\langle n\left(\mathbf{r}^{\prime}, 0\right) n\left(\mathbf{r}+\mathbf{r}^{\prime}, t\right)\right\rangle
$$

where we have assumed that the system is invariant under time translation operations. The frequency component $\omega$ of this function is the density correlation function $p_{E}(\mathbf{r})$ of an excitation of energy $E=\hbar \omega$ in the system

$$
p_{E}(\mathbf{r})=F T_{t}[p(\mathbf{r}, t)]
$$

Here and in the following we use the abbreviation $F T_{x}[f(x)]$ for the Fourier transform of a function with respect to the variable $x$.

Applying the Wiener-Khinchin theorem, $p_{E}(\mathbf{r})$ is the Fourier transform of the dynamic form factor(see e. g. [6]) $S(\mathbf{q}, E)$ of the scatterer

$$
p_{E}(\mathbf{r})=F T_{\mathbf{q}}[S(\mathbf{q}, E)]
$$

Experimentally, the inelastic electron scattering cross section for cubic systems is isotropic and to a good approximation a Lorentzian in wave number transfer $q$ with cutoff $q_{c}$. As we shall see in the following the dynamic form factor must then be proportional to $q^{2}$ up to the cutoff and vanish thereafter.

In this approximation the Fourier transform Eq. 3 can be performed directly

$$
\begin{aligned}
p_{E}(r) & =2 \pi \int_{q_{e}}^{q_{c}} q^{2} S(q, E) d q \int_{0}^{\pi} d \vartheta \sin (\vartheta) e^{i q r \cos (\vartheta)} \\
& =4 \pi \int_{q_{e}}^{q_{c}} q^{4} \frac{\sin (q r)}{q r} d q
\end{aligned}
$$

where we have used the fact that no wave number transfer less than the characteristic transfer $q_{e}=k_{0} E / 2 E_{0}$ can occur.

In the electron microscope, spatial information is only available projected onto the image plane with coordinates $\mathbf{x}$ (which corresponds to the subspace in reciprocal space subtended by the diffraction plane). When comparing predictions with experimental results one is rather interested in the z-projected correlation function $\bar{p}=\int p(\mathbf{r}) d z$. This is the two-dimensional Fourier transform with respect to the coordinates $\mathbf{q}$ in the diffraction plane. In dipole approximation with cutoff $q_{c}$ this is [7]

$$
\begin{aligned}
\bar{p}_{E}(\mathbf{x}) & =F T_{\mathbf{q}}[S(\mathbf{q}, E)]=\int_{0}^{q_{c}} S(q, E) e^{i \mathbf{q} \mathbf{x}} d^{2} q \\
& =2 \pi \int_{0}^{q_{c}} S(q, E) e^{i q x \cos (\varphi)} q d q d \varphi \\
& =2 \pi \int_{0}^{q_{c}}\left(q_{e}^{2}+q^{2}\right) J_{0}(q x) q d q .
\end{aligned}
$$

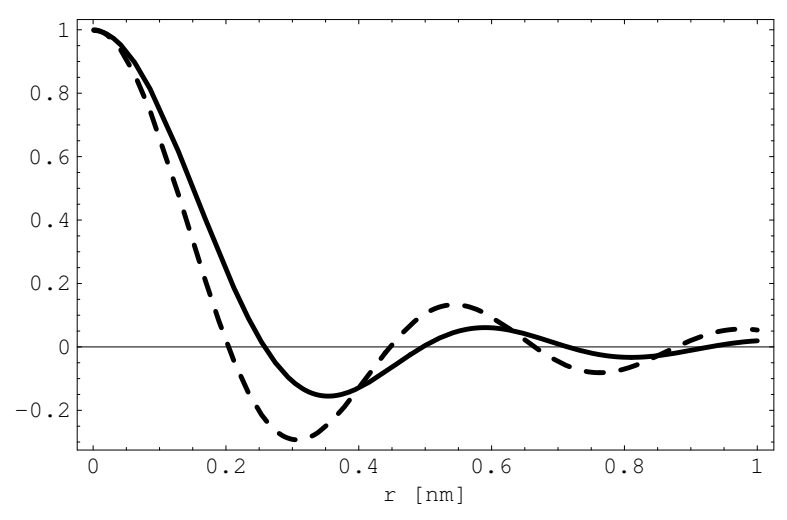

FIG. 1: Density autocorrelation function of Al plasma excitation. Full line: Eq. 4, dashed line: projection onto plane of observation, Eq 5.

Results are shown for Al, compared to the 3dimensional correlation function, in Fig. 1. The 3D correlation function has ist first zero at $0.25 \mathrm{~nm}$ and ist first subsidiary minimum at $0.35 \mathrm{~nm}$ with less than $20 \%$ probability.

\section{VARIOUS APPROACHES TO CORRELATION}

A basic reasoning suggests that the correlation length is of the same magnitude as the extension of the exchange-correlation hole[8]. In metals, both these lengths estimate to the inter-electron distance, $i$. e. some Ångstroms.

This is consistent with the findings of Egri [9, 10] who derived an extension of $\approx 0.2 \mathrm{~nm}$ for the plasmon in metals and semiconductors from the analysis of what he termed the plasmon wave function. The same order of magnitude is found for the pair distribution function in a coherent superposition of electron-hole excitations[11]. A classical argument suggests to define the extension of the plasmon as the product of the lifetime and the group velocity. For Al, this was found to be $0.4 \mathrm{~nm}[12]$. This last value seems to be in agreement with early experiments on the spatial resolution in plasmon filtered images[13].

However, more recently Lichte and coworkers[14-16] reported interference experiments with fast electrons having excited a plasmon in $\mathrm{Al}$. Contrary to the predictions they found a coherence length of almost $10 \mathrm{~nm}$, lateron $30 \mathrm{~nm}$ from fringe contrast in the inelastic hologram.

This seems to be contradicted by the uncertainty principle: the coherent extension of a plasmon scattered electron in the electron microscope is by definition $\delta x=$ $\hbar / \sqrt{\left\langle\delta q^{2}\right\rangle}$. Since the angular scattering distribution is proportional to a Lorentzian $\left(q_{e}^{2}+q^{2}\right)^{-1}$ the variance of which is infinite the coherence length of the scattered electron comes out as $\delta x=0$.

In the following we discuss the various approaches more 
precisely. We shall se that the intuitive argument based on the angular scattering distribution is not far from reality.

\section{A. Mean free path of the excited plasmon}

Cheng[12] calculated what he called the localization distance of the excitation as the product of group velocity with the relaxation time. The former was taken to be an average over the observable wave numbers, and the latter was obtained from the energy FWHM of the plasmon peak. The result was $0.4 \mathrm{~nm}$. In fact, what was computed was the mean free path of the plasmon. This need not be the correlation length as becomes evident when one thinks of solitons which travel over much longer distances than their spatial extension. In this paper, the puzzling observation can be found that from the angular distribution of scattering a localization distance of $23 \mathrm{~nm}$ can be derived [35], in disagreement with experimentally observed spatial resolution of $0.4 \mathrm{~nm}$. The situation is confusing since three different quantities are compared there. Intuitively it is felt that they are related to each other in some way, but how has not been clear.

\section{B. Exchange hole}

In the ground state of a free non-interacting gas of $N$ electrons of volume $v$, the pair distribution function of particles is

$$
p(\mathbf{r})=\delta^{3}(\mathbf{r})+(N-1) / v .
$$

In the Hartree-Fock approximation we see a decrease in $p$ in the vicinity of $r=0$ known as exchange hole[8]

$$
g(r)=-\frac{9\left(-\left(k_{F} r \cos \left(k_{F} r\right)\right)+\sin \left(k_{F} r\right)\right)^{2}}{2 k_{F} r^{6}}
$$

$g$ is a universal function of the dimensionless variable $r k_{F}$ where $k_{F}$ is the Fermi wave number. The density correlation is now

$$
p(\mathbf{r})=\delta^{3}(\mathbf{r})+\frac{(N-1)}{v}\left(1-g\left(k_{F} r\right)\right) .
$$

This hole describes that electrons try to avoid each other according to the Pauli principle. When, additionally, the electrons are allowed to interact the Coulomb repulsion will increase the extension of the hole[8]. The size of this repulsion area around an electron gives a clue to the correlation length of electrons in the excited state. It turns out to be close to the screening length for electrostatic perturbations which in turn equals roughly the average distance of electrons in the gas. For Al, this is $0.107 \mathrm{~nm}$, and we find a FWHM of this hole of $0.1 \mathrm{~nm}$ and the first zero is at $0.23 \mathrm{~nm}$.

\begin{tabular}{|l||c|c|c|}
\hline method & HWHM & 1. zero & 1. minimum \\
\hline$p_{E}(x)$ & 0.13 & 0.20 & 0.30 \\
$p_{E}(r)$ & 0.16 & 0.25 & 0.36 \\
exchange hole & 0.10 & 0.23 & 0.23 \\
pair distribution & 0.16 & 0.28 & 0.38 \\
electron-hole distance & 0.20 & 0.35 & 0.46 \\
\hline
\end{tabular}

TABLE I: Electron correlation in the Al plasma. The table lists several parameters of the distribution functions (values in $\mathrm{nm}$ ) as defined in the text.

\section{Pair distribution function in correlated electron-hole excitations}

In 1957 Ferrell and Quinn[11] explained the high energy of the plasma excitation in a metal with the positional correlation of electrons in the excited state. These correlations stem from coherent superpositions of excited single-electron states with the same relative phase. The pair distribution function of electrons in this excited state with wave vector $\mathbf{k}$ is $\propto \cos (\mathbf{k x})$. The pair distribution function is the same as the density-density correlation function. Averaging over directions

$$
p(r)=\frac{\sin (k r)}{k r}
$$

For $k$ we may take the mean wave number $\bar{k}$ of a plasma excitation. Assuming that all $q$ values from 0 to the cutoff wave number $q_{c}$ are equiprobable we obtain $\bar{k}=3 q_{c} / 4$. The FWHM is at $0.16 \mathrm{~nm}$, the first zero at $0.28 \mathrm{~nm}$.

\section{Electron-hole distance}

An alternative approach to the correlation length starts from the plasmon wave function[9], defined as the probability amplitude of finding electron-hole pairs separated by r. In a way, this is the opposite of Ferrell's approach for, if the probability to find two electrons at a distance is high, there will be a low chance to find a hole at the same distance. The result for small $k$ and $\mathbf{r}$ perpendicular to $\mathbf{k}$ is

$$
\varphi\left(k_{F} r\right)=\frac{2 J_{1}\left(k_{F} r\right)}{k_{F} r}
$$

For $\mathbf{r}$ parallel to $\mathbf{k}$ the extension of the wave function is bigger by a factor of roughly 2.2. Averaging over both values, the resulting FWHM is $0.14 \mathrm{~nm}$, and the first zero is at $0.35 \mathrm{~nm}$.

Several values for the extension of the plasma excitation in $\mathrm{Al}$ metal are collected in Table 1. It can be seen that the various approaches yield very similar values both for the HWHM and for the zeros. 


\section{INELASTIC ELECTRON SCATTERING}

In the single scattering approximation, the dynamic form factor $S$ of a scattering medium relates to the double differential inelastic scattering cross section for fast electrons as $[6]$

$$
\frac{\partial^{2} \sigma}{\partial E \partial \Omega}=\frac{4 \gamma^{2}}{a_{0}^{2}} \frac{k}{k_{0}} \frac{S(\mathbf{Q}, E)}{Q^{4}},
$$

where $a_{0}=4 \pi \varepsilon_{0} \hbar^{2} / m e^{2}$ is the Bohr radius, and $\gamma$ is a relativistic factor. We used the 3 -dimensional vector in reciprocal space

$$
\mathbf{Q}=\left(\begin{array}{c}
\mathbf{q} \\
q_{E}
\end{array}\right)
$$

$q_{E}=k-k_{0}$ is the negative difference of radii of the Ewald spheres before and after inelastic scattering. For small energy losses $E<<E_{0}$ we have approximately $q_{E}=-k_{0} E / 2 E_{0}$. Eq. 11 is valid in the first Born approximation for an incident plane wave, and for single scattering.

Experimentally, the angular scattering distribution is well approximated by a Lorentzian behaviour up to a cutoff angle $\theta_{c}$. Therefore the dynamic form factor in Eq. 11 must be proportional to $Q^{2}$ up to $\sqrt{q_{E}^{2}+k_{0}^{2} \theta_{c}^{2}}$. This is the dipole approximation which is often used for ionisation edges[7].

There is an intimate relationship between the double differential scattering cross section Eq. 11 and the density matrix $\rho_{E}\left(\mathbf{q}, \mathbf{q}^{\prime}\right)$ of the probe electron after inelastic scattering with energy loss $E$ in the diffraction plane: the former is proportional to the diagonal element of the latter.

The propagation of the electron's density matrix is described by the kinetic equation[17]. When Bragg scattering can be neglected and the specimen is thinner than a mean free path for inelastic scattering (which is roughly $100 \mathrm{~nm}$ for $200 \mathrm{keV}$ incident energy) the single scattering approximation applies[7], and we obtain

$$
\rho_{E}\left(\mathbf{q}, \mathbf{q}^{\prime}\right)=\left(\frac{m e^{2} \pi}{\varepsilon_{0} \hbar^{2} k}\right)^{2} \frac{S\left(\mathbf{Q}, \mathbf{Q}^{\prime}, E\right)}{Q^{2} Q^{\prime 2}} .
$$

The quantity $S\left(\mathbf{Q}, \mathbf{Q}^{\prime}, E\right)$ on the right hand side is the mixed dynamic form factor[18]. Eq. 11 is a special case of eq. 12 for $\mathbf{q}=\mathbf{q}^{\prime}$. The density matrix of the inelastically scattered electron in real space is the Fourier transform of eq. 12

$$
\rho_{E}\left(\mathbf{x}, \mathbf{x}^{\prime}\right)=F T_{\mathbf{q}, \mathbf{q}^{\prime}}\left[\rho_{E}\left(\mathbf{q}, \mathbf{q}^{\prime}\right)\right]
$$

and therein we have all available information on the probe - localisation and coherence. The Fourier transform is the basis for the close connection between the mixed dynamic form factor and channeling phenomena in inelastic scattering $[19,20]$.

Via eq.3 and 12 we have experimental access to the correlation function, measuring the inelastic differential cross section. This is however problematic since the Fourier transform of the Coulomb interaction $1 / q^{4}$ between probe and scatterer acts as a low pass filter. Noise and multiple scattering in the specimen will introduce considerable errors at higher $q$. In order to bypass this obstacle it has been tried to obtain information on the correlation of the elementary excitations from holography experiments[14-16].

\section{A. Electron Holography}

Electron holography is based on electron interference. The first electron interferometer used amplitude splitting at crystals both for splitting and superimposing the electron waves[21]. A crystal can be considered an amplitude splitter in that, by diffraction, the incoming wave is split up into partial waves, which are superimposed subsequently. Lateron, this principle was also used by others[22-24].

In todays holography, however, the principle of wavefront splitting using the Mllenstedt electron biprism[25] is most powerful as a beam splitter. A biprism is a wavefront splitter, splitting each wave $\psi(\vec{x})$ emerging from the object plane into two partial waves which are subsequently superimposed in the detector plane with a relative displacement (shear) $\vec{d}$. For an overview see e.g. [26]

We assume here and in the following that the lens system is aberration free, which is reasonable, since the experiments to be discussed are far from atomic resolution. Assume that a single exit wave $\psi(\vec{x})$ is defined over the entire object plane. The biprism will induce a relative shift $\mathbf{d}$ between two partial images. Consequently, in the detector plane the wave function is a superposition of the two partial waves emerging at $\mathbf{x}-\mathbf{d} / 2$ ) and at $\mathbf{x}+\mathbf{d} / 2$ )

$$
\psi(\mathbf{x})=\psi(\mathbf{x}-\mathbf{d} / 2) e^{i \mathbf{k}_{c} / 2 \mathbf{x}}+\psi(\mathbf{x}+\mathbf{d} / 2) e^{-i \mathbf{k}_{c} / 2 \mathbf{x}}
$$

where $\mathbf{k}_{c}=k \beta \mathbf{e}_{d}$ is the carrier frequency of the interference fringes proportional to the superposition angle $\pm \beta$ induced by the biprism to the two partial waves. The unit vector $\mathbf{e}_{d}$ is perpendicular to the axis of the biprism (parallel to $\mathbf{d}$ ).

After squaring one finds the stationary interference pattern

$$
\begin{aligned}
I(\mathbf{x})= & I(\mathbf{x}-\mathbf{d} / 2)+I(\mathbf{x}+\mathbf{d} / 2)+ \\
& 2 \Re\left[\psi(\mathbf{x}-\mathbf{d} / 2) \psi^{*}(\mathbf{x}+\mathbf{d} / 2) e^{i \mathbf{k}_{c} \mathbf{x}}\right]
\end{aligned}
$$

The last (interference) term can be written alternatively

$$
2\left|\psi(\mathbf{x}-\mathbf{d} / 2) \psi^{*}(\mathbf{x}+\mathbf{d} / 2)\right| \cos \left(\mathbf{k}_{c} \mathbf{x}+\Delta \phi(\mathbf{x}, \mathbf{d})\right) .
$$

Here, $\Delta \phi$ is the phase shift between waves emerging at points $\mathbf{x}-\mathbf{d} / 2$ and $\mathbf{x}+\mathbf{d} / 2$. 


\section{B. Partially coherent illumination}

We assume now that the object is illuminated by a monochromatic, extended source. A point at $r$ s in the source contributes intensity $i_{s}(\mathbf{r s})$, and induces an additional phase shift $e^{i \gamma(\mathbf{r s}, \mathbf{x})}$ to the object exit wave where $\gamma$ depends on the action taken between source and object[36]. When we integrate over $\mathbf{r}$ in the correspondingly changed eq.16, the intensity terms remain unchanged since the phases $\gamma$ cancel, and the interference term becomes

$$
2 \Re\left[\psi^{*}(\mathbf{x}-\mathbf{d} / 2) \psi(\mathbf{x}+\mathbf{d} / 2) e^{-i \mathbf{k}_{c} \mathbf{x}} \mu_{s c}(\mathbf{d})\right] .
$$

where we have used the fact that the integral over the source illumination function is(rs)is the spatial coherence $\mu_{s c}$

$$
\mu_{s c}(\mathbf{d})=\int i_{s}(\mathbf{r s}) e^{i(\gamma(\mathbf{r s}, \mathbf{x}+\mathbf{d} / 2)-\gamma(\mathbf{r s}, \mathbf{x}-\mathbf{d} / 2))} d^{2} r s .
$$

In the far field approximation, the Fresnel propagator degenerates to the Fourier transform, and $\mu_{s c}(d)=$ $F T_{\vec{r}_{s}}\left[i_{s}\right]$. Usually, in electron microscopy the source is assumed to be rotationally symmetric; hence $\mu_{s c}=\mu_{s c}(d)$ is real. More generally, the partial coherence function should also include temporal coherence $\mu_{t c}(n)$ with $n$ the order of interference however, usually the wave number spectrum is so narrow that temporal coherence need not be considered here. In this case we can write

$$
\begin{gathered}
I(\mathbf{x})=I(\mathbf{x}-\mathbf{d} / 2)+I(\mathbf{x}+\mathbf{d} / 2)+ \\
2 \mu_{s c}(d)\left|\psi(\mathbf{x}-\mathbf{d} / 2) \psi^{*}(\mathbf{x}+\mathbf{d} / 2)\right| \cos \left(\mathbf{k}_{c} \mathbf{x}+\Delta \phi(\mathbf{x}, \mathbf{d})\right)
\end{gathered}
$$

The two superimposed waves have a degree of spatial coherence $\mu_{s c}$. Both $\beta$ and shear $d$ are proportional to the applied biprism voltage with a proportionality factor given by the geometry of the setup. Spatial coherence of illumination can be determined by measuring the contrast for different shear (i.e. changing the biprism voltage)[27]. remark: you need the FT only, if you want to determine the source function is (rs), which is not our interest here. It may be used later, if we want to determine the coherence function in Fourier space (diffraction pattern).

If one partial wave runs entirely through vacuum and the other one through an object, the elastic interaction (without any energy transfer) in general results in a modulation of amplitude and phase with respect to vacuum. The first partial wave is then given as $\psi_{v a c}=1$ and the second one as $\left|\psi_{o b j}\right| e^{i \Delta \phi}$. Assuming that the specimen occupies the positive half space of the object plane (its edge passing through $\vec{x}=0$, perpendicular to $\vec{d})$, the interferogram eq. 17 reads

$$
\begin{aligned}
I(\mathbf{x})= & 1+I_{o b j}(\mathbf{x}+\mathbf{d} / 2)+ \\
& 2 \mu_{s c}(\vec{d})\left|\psi_{o b j}(\mathbf{x}+\mathbf{d} / 2)\right| \cos \left(\mathbf{k}_{c} \mathbf{x}+\Delta \phi(\mathbf{x}, \mathbf{d} / 2)\right)
\end{aligned}
$$

where the mean fringe contrast is still given by the degree of coherence of illumination. Such interferograms give rise to all sorts of applications in electron interferometry and holography; for a review see[26].

\section{Inelastic Holography}

It has been shown[28] that the interference fringes produced by superposition of waves inelastically scattered with energy loss $\delta E$ with the vacuum reference wave disappear in the record, when the recording time obeys

$$
\tau>h / \delta E
$$

Since $\tau$ is of the order of seconds, the limiting energy loss is about $10^{-15} \mathrm{eV}$. In a way, the biprism is a perfect energy filter.

The fringes disappear, because they are not stationary but run with a velocity proportional to the energy difference between the two partial waves. If both waves would have suffered the same energy loss, the fringes reappear only, if phase coherence is given, i.e. if the phases are correlated. In order to study phase correlation, it is therefore reasonable to bring two waves with the same energy loss from different positions in the specimen to interference. Since elastic holography detects phase relations between different points, it can be anticipated that the same is true for inelastic holography. Any non-arbitrary phase relation between different points of excitation could be detected. This would then be an elegant pathway to study the correlation in solid state excitations. Since it has been argued that ionisation is a very localized process, one would not expect correlations between points at a larger distance than the atomic scale. On the other hand, plasma excitations seem a good candidate for inelastic holography since they are collective throughout the medium. The decisive criterion is then, whether the suffered interaction processes preserve phase coherence between the two waves.

Experimentally, the question was tackled in an electron microscope equipped with an energy filter. Both waves were run through adjacent areas of the same object hence offering the same inelastic interactions to the beam electrons. Indeed, interference fringes with sufficient contrast were found when selecting the Al-plasmon loss by the energy filter[14, 15]. In the latter experiment, by means of the biprism voltage, the lateral distance $d$ (shear) was made as large as $10 \mathrm{~nm}$ without loosing the contrast in electron noise. This means that the radius of a coherence patch in the outgoing wavefield is at least $10 \mathrm{~nm}$ wide.

What is measured in this experiment is the mutual coherence function[29] of electrons in the exit plane of the specimen having suffered a plasmon loss.

In analogy to the elastic case eq. 16 one could argue that the hologram reads

$$
\begin{aligned}
I(\mathbf{x})= & I(\mathbf{x}-\mathbf{d} / 2)+I(\mathbf{x}+\mathbf{d} / 2)+ \\
& 2 \mu_{s c}(\mathbf{d}) \Re\left[\psi_{E}(\mathbf{x}-\mathbf{d} / 2) \psi_{E}^{*}(\mathbf{x}+\mathbf{d} / 2) e^{i \mathbf{k}_{c} \mathbf{x}}\right]
\end{aligned}
$$

The essential question is whether the relative phase between the two partial interfering waves $\psi_{E}$ of inelastically scattered electrons at positions $\mathbf{x}-\mathbf{d} / 2$ and $\mathbf{x}+\mathbf{d} / 2$ is fixed as in the elastic case, or arbitrary. If it is arbitrary, 
it would induce a new kind of (inelastic) incoherence. In this case it would be necessary to average the interference term in eq. 19 in order to write

$I(\mathbf{x})=I(\mathbf{x}-\mathbf{d} / 2)+I(\mathbf{x}+\mathbf{d} / 2)+2 \mu_{\text {out }}(\mathbf{d}) \cos \left(\mathbf{k}_{c} \mathbf{x}+\Delta \phi(\mathbf{x}, \mathbf{d})\right.$

with the mutual coherence function of the outgoing electrons

$$
\mu_{\text {out }}(\mathbf{d})=\mu_{s c}(\mathbf{d})\left\langle\psi_{E}(\mathbf{x}-\mathbf{d} / 2) \psi_{E}^{*}(\mathbf{x}+\mathbf{d} / 2)\right\rangle
$$

It is not quite clear how the wave function after inelastic scattering should enter this averaging process, and how the phase shift $\Delta \phi(\mathbf{x}, \mathbf{d})$ should be obtained.

A closer inspection shows that this is indeed the weak point because, after inelastic interaction with energy loss $\mathrm{E}$, the probe electron is not in a pure state any longer. Rather, it is entangled with the specimen's electrons. This means that it can no longer be described by a wave function.

The proper tool for describing mixed states is the density matrix [37]. We build the intensity of the mixed state as the sum over the individual exit wave functions which contribute to a given energy loss, with eq.14:

$$
\begin{aligned}
I_{E}(\mathbf{x})= & \sum_{j}\left|\psi_{j}(\mathbf{x}-\mathbf{d} / 2)\right|^{2}+\sum_{j}\left|\psi_{j}(\mathbf{x}+\mathbf{d} / 2)\right|^{2} \\
& 2 \Re\left[\sum_{j} \psi_{j}(\mathbf{x}-\mathbf{d} / 2) \psi_{j}^{*}(\mathbf{x}+\mathbf{d} / 2) e^{i \mathbf{k}_{c} \mathbf{x}}\right] .
\end{aligned}
$$

With the substitutions

$$
\begin{aligned}
& \overline{\mathbf{x}}=\frac{\mathrm{x}+\mathrm{x}^{\prime}}{2} \\
& \mathbf{d}=\mathbf{x}-\mathrm{x}^{\prime}
\end{aligned}
$$

and the inelastic density matrix for probe electrons after energy loss $E$

$$
\rho_{E}\left(\mathbf{x}, \mathbf{x}^{\prime}\right)=\sum_{j} \psi_{j}(\mathbf{x}) \psi_{j}^{*}\left(\mathbf{x}^{\prime}\right)
$$

this can be written as

$$
I_{E}(\overline{\mathbf{x}})=\rho_{E}(\mathbf{x}, \mathbf{x})+\rho_{E}\left(\mathbf{x}^{\prime}, \mathbf{x}^{\prime}\right)+2 \Re\left[\rho_{E}\left(\mathbf{x}, \mathbf{x}^{\prime}\right) e^{i \mathbf{k}_{c} \overline{\mathbf{x}}}\right] .
$$

The coherence of the incident wave field can be included exactly as in eq.17 for the elastic case, integrating over the source area. Eq. 20 is then

$$
I_{E}(\overline{\mathbf{x}})=\rho_{E}(\mathbf{x}, \mathbf{x})+\rho_{E}\left(\mathbf{x}^{\prime}, \mathbf{x}^{\prime}\right)+2 \mu_{s c}(\mathbf{d}) \Re\left[\rho_{\mathbf{E}}\left(\mathbf{x}, \mathbf{x}^{\prime}\right) \mathbf{e}^{\mathbf{i} \mathbf{k}_{\mathbf{c}} \overline{\mathbf{x}}}\right] .
$$

For a pure state, eq. 20 would simplify to eq. 19. Indeed, the difference is in the interference term which is now an off-diagonal element of the density matrix, multiplied with the carrier frequency and the coherence of the incident wave field.[38] This is the fundamental equation. It is a natural extension of the pure state description to an arbitrary number of (incoherently) superposed wave functions, and is as such well adapted to the case of inelastic scattering.
For isotropic scattering, the density matrix $\rho_{E}$ is real, so

$$
I_{E}(\overline{\mathbf{x}})=\rho_{E}(\mathbf{x}, \mathbf{x})+\rho_{E}\left(\mathbf{x}^{\prime}, \mathbf{x}^{\prime}\right)+2 \mu_{s c}(\mathbf{d}) \rho_{\mathbf{E}}\left(\mathbf{x}, \mathbf{x}^{\prime}\right) \cos \left(\mathbf{k}_{\mathbf{c}} \overline{\mathbf{x}}\right) .
$$

We define the mutual coherence function of the outgoing wave field as

$$
\mu_{\text {out }}\left(\mathbf{x}, \mathbf{x}^{\prime}\right)=\mu_{s c}\left(\mathbf{x}-\mathbf{x}^{\prime}\right) \rho_{E}\left(\mathbf{x}, \mathbf{x}^{\prime}\right) .
$$

(when we consider these quantities as matrices this is the "dot" product of the incident electron's coherence and the inelastic density matrix) and write eq. 22

$$
I_{E}(\mathbf{x})=\rho_{E}(\mathbf{x}, \mathbf{x})+\rho_{E}\left(\mathbf{x}^{\prime}, \mathbf{x}^{\prime}\right)+2 \mu_{\text {out }}\left(\mathbf{x}, \mathbf{x}^{\prime}\right) \cos \left(\mathbf{k}_{c} \overline{\mathbf{x}}\right) .
$$

The solid state plasma can be considered as homogeneous and isotropic. In this case, the density matrix in Eq. 22 depends only on the difference $|\mathbf{d}|$ of the two variables. (the main diagonal value, $i$. $e$. the density is constant, $\rho_{E}(\mathbf{x}, \mathbf{x})=\rho_{E}(0,0)$.) The mutual coherence function eq.23 simplifies to

$$
\mu_{\text {out }}(d)=\mu_{s c}(d) \rho_{E}(0, d)
$$

and the measured intensity is

$$
I_{E}(\mathbf{x})=2 \rho_{E}(0,0)+2 \mu_{\text {out }}(d) \cos \left(\mathbf{k}_{c} \mathbf{x}\right) .
$$

The amplitude of the fringes measures the off-diagonal element of the inelastic density matrix $\rho_{E}(0, d)$, which, in turn is identical to the mutual object transparency for energy loss $E$, introduced by Rose[30]. A good description of image formation both for potential scattering and for inelastic interactions, based on the mutual coherence function and mutual object transparency is the treatment of Müller et al.[31]. The fringe contrast in the inelastic hologram is given in terms of the mutual object transparency $\mu_{\text {out }}$ as

$$
\frac{I_{\max }-I_{\min }}{I_{\max }+I_{\min }}=\frac{\mu_{\text {out }}}{\rho_{E}}
$$

In complete analogy to potential scattering, we can then relate the fringe contrast to the mutual coherence function. Therefrom, the off-diagonal term of the density matrix of the inelastically scattered electron can be easily obtained from a hologram.

Predicting fringe contrast needs the calculation of $\rho_{E}(0, d)$. Quite generally, for an arrangement of scatterers with spatial distribution $g(\mathbf{r})$ the total density matrix is

$$
\rho_{E}^{t}\left(\mathbf{x}, \mathbf{x}^{\prime}\right)=\int g(\mathbf{r}) \rho_{E}\left(\mathbf{x}-\mathbf{r}, \mathbf{x}^{\prime}-\mathbf{r}\right) d^{2} r
$$

For a homogeneous infinitely extended system we use the mixed dynamic form factor obtained by integrating over all independent scattering centres at $\mathbf{r}$ with proper phase shifts:

$$
S^{h}\left(\mathbf{Q}, \mathbf{Q}^{\prime}, E\right)=\int \mathbf{Q} \cdot \mathbf{Q}^{\prime} e^{i\left(\mathbf{q}-\mathbf{q}^{\prime}\right) \mathbf{r}} d^{2} r=\delta^{2}\left(\mathbf{q}-\mathbf{q}^{\prime}\right) .
$$




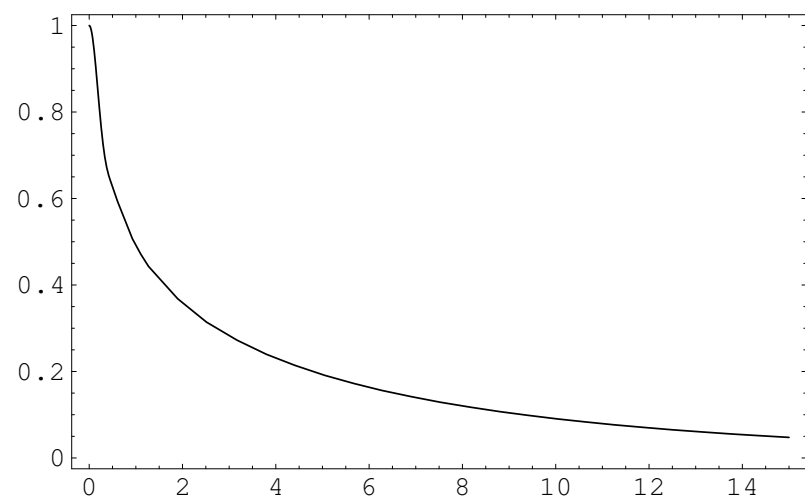

FIG. 2: Off-diagonal density matrix element (mutual object transparency) $\rho_{E}^{h}(0, d)$ of electrons emerging from the exit plane of an infinitely extended homogeneous Al specimen, after excitation of a plasmon, as a function of holographic shear.

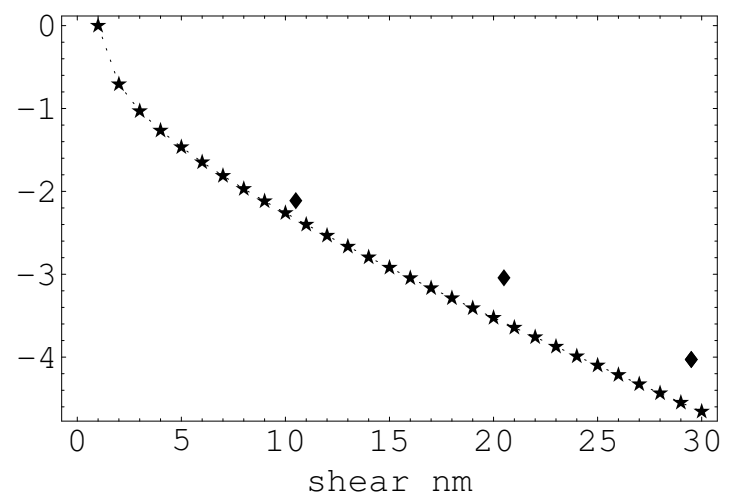

FIG. 3: Same as in fig. 2 on a logarithmic scale, compared with experimental values[33].

The corresponding density matrix is obtained as the Fourier transform of eq. 27

$$
\begin{aligned}
\rho_{E}^{h}\left(\mathbf{x}, \mathbf{x}^{\prime}\right) & =F T_{\mathbf{q}, \mathbf{q}^{\prime}}\left[\frac{S^{h}\left(\mathbf{Q}, \mathbf{Q}^{\prime}, E\right)}{Q^{2} Q^{\prime 2}}\right] \\
& =\int_{0}^{q_{c}} Q^{-2} e^{i \mathbf{q}\left(\mathbf{x}-\mathbf{x}^{\prime}\right)} \doteq K_{0}^{t}\left(q_{e}\left(\left|\mathbf{x}-\mathbf{x}^{\prime}\right|\right)\right)
\end{aligned}
$$

The last integral was approximated by the truncated Bessel function which we define in terms of the Bessel function of second kind and zeroth order $K_{0}$ as

$$
K_{0}^{t}(x)= \begin{cases}1 & x<1 / q_{c} \\ \frac{K_{0}\left(q_{E} x\right)}{\ln \left(q_{c} / q_{E}\right)} & \text { else. }\end{cases}
$$

The function $\rho_{E}^{h}(0, d)$ is shown in Fig. 2 for the Al plasmon. It can be interpreted as the contrast of fringes as a function of shear $d$ for perfectly coherent illumination[32]. The $10 \%$ level is reached at a shear of $9.1 \mathrm{~nm}$, and at $20 \mathrm{~nm}$ we find 0.026 , both in good agreement with the interference experiments of Lichte and coworkers[15, 16].

We can now compare the mutual object transparency with the correlation function in the Al plasmon, Eq. 5.

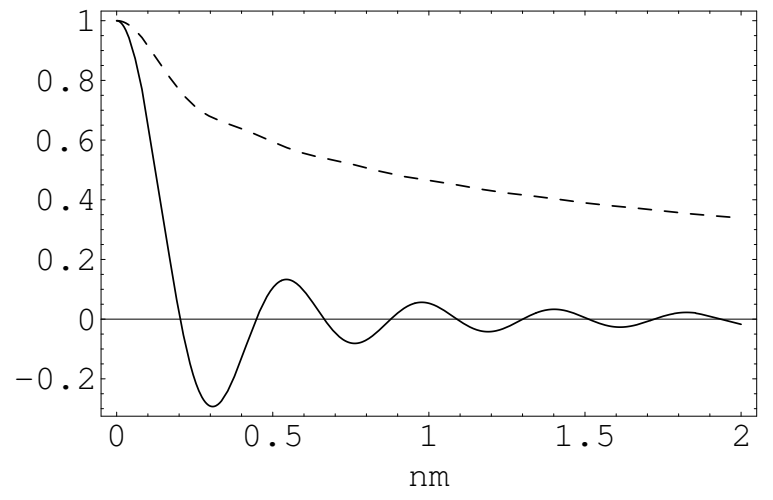

FIG. 4: Dashed line: Off-diagonal density matrix element (mutual object transparency) $\rho_{E}^{h}(0, d)$ of electrons, compared with the 2-dimensional projection of the density correlation function of the scatterer, Eq 5 (full line) as a function of shear.

Fig. 4 shows the first $2 \mathrm{~nm}$ together with the 2-D correlation function. The mutual coherence function is smoothly decreasing; it is so broad because of the long range Coulomb interaction between target charges and the probe electron. The faint wiggles at the first few $\mathrm{nm}$ are remnants of the strong oscillations in the correlation function.

\section{PROPOSAL FOR EXPERIMENTS}

In the following we apply the outlined theory in two cases, using the inelastic density matrix for the calculation of a) the inelastic mutual coherence outside an excited medium; and b) we predict reversal of the fringe contrast in the vicinity of a small isolated area of excitation. The first result is unexpected and could be tested by holography. The second result is even more surprising because it would show, for the first time directly, a phase shift of $\pi$ in inelastic scattering. [39].

\section{A. Plasmon coherence in vacuum}

It has been shown[7] that the intensity of a pointlike scatterer decays as $K_{0}^{t}\left(q_{e} x\right)^{2}$ with distance from the excitation. Since the coherence function decays as $K_{0}^{t}\left(q_{e} x\right)$ one might expect unusual behaviour of the holographic fringe contrast in the vicinity of the edge of a specimen.

The total density matrix for a distribution of scatterers filling the half space $\mathbf{r}=(\xi>0, \eta)$ is

$$
\rho_{E}^{t}\left(\mathbf{x}, \mathbf{x}^{\prime}\right)=\int_{-\infty}^{\infty} \int_{0}^{\infty} g(\mathbf{r}) \rho_{E}\left(\mathbf{x}-\mathbf{r}, \mathbf{x}^{\prime}-\mathbf{r}\right) d \xi d \eta
$$

Numerical integration yields the intensity profile and the coherence function as shown in Fig. 5 .

Note the decrease in the mutual object transparency close to the border of the specimen. This is a consequence 


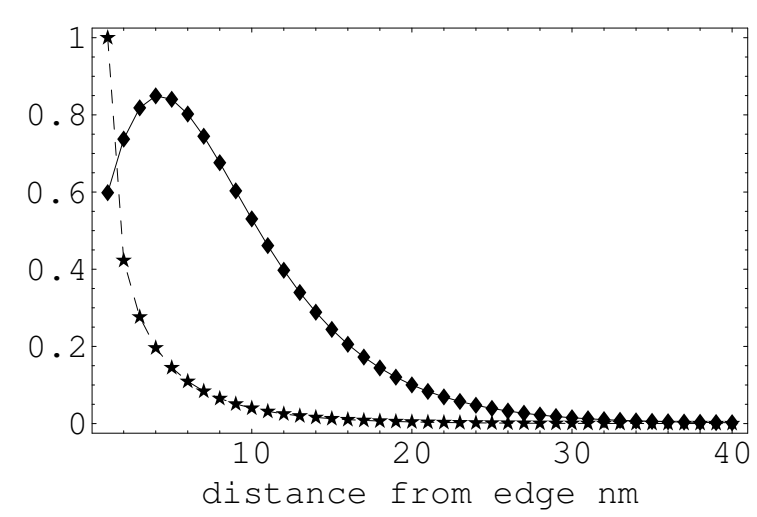

FIG. 5: Intensity (dashed) and mutual object transparency $\rho_{E}^{h}(\mathbf{x}, \mathbf{x}+\mathbf{d})(\diamond)$ outside of an $\mathrm{Al}$ specimen as a function of distance from the border. The shear is $20 \mathrm{~nm}$ parallel to the edge of the specimen. Both curves are normalized to intensity 1 at the edge. The mutual object transparency is enlarged by a factor of 50 .

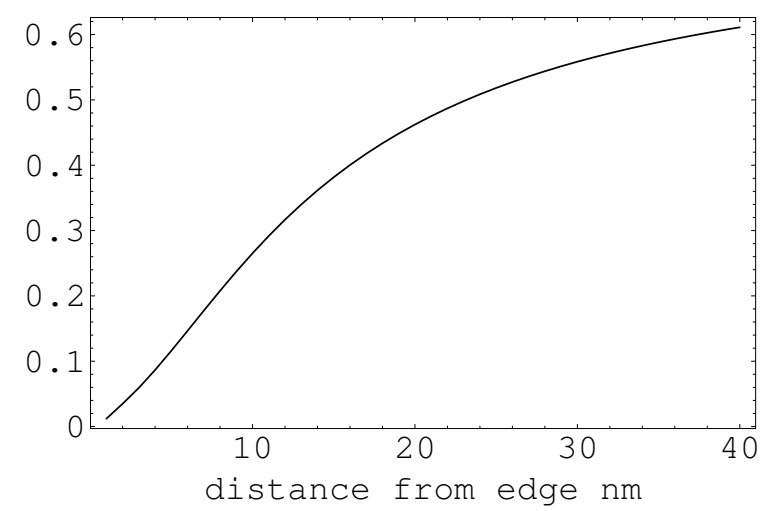

FIG. 6: Fringe contrast outside of $\mathrm{Al}$ specimen for a shear of $20 \mathrm{~nm}$ parallel to the edge of the specimen.

of the negative contributions to the density matrix for distances that are small with respect to the shear.

The fringe contrast eq. 26 is shown in fig. 6 as a function of distance from the edge. It is seen that the contrast increases outside the scattering medium. This surprising behaviour can be attributed to the faster decrease of the intensity (which behaves approximately as $K_{0}^{2}$ ) with respect to the coherence function. At the very edge of the specimen, the contrast is 0.012 whereas in the medium (far from the edge) eq. 29 predicts 0.025 . That means that we should see a small dip in contrast when we approach the specimen's border from the inside.

\section{B. Contrast reversal in small excited areas}

Assume a pointlike scatterer. When we impose a shear $d$ by the biprism we will see the source of inelastic scattering overlapping with the shifted second image. Since the intensity decreases smoothly outside the inelastic source and its image, we will also detect the tails of the second

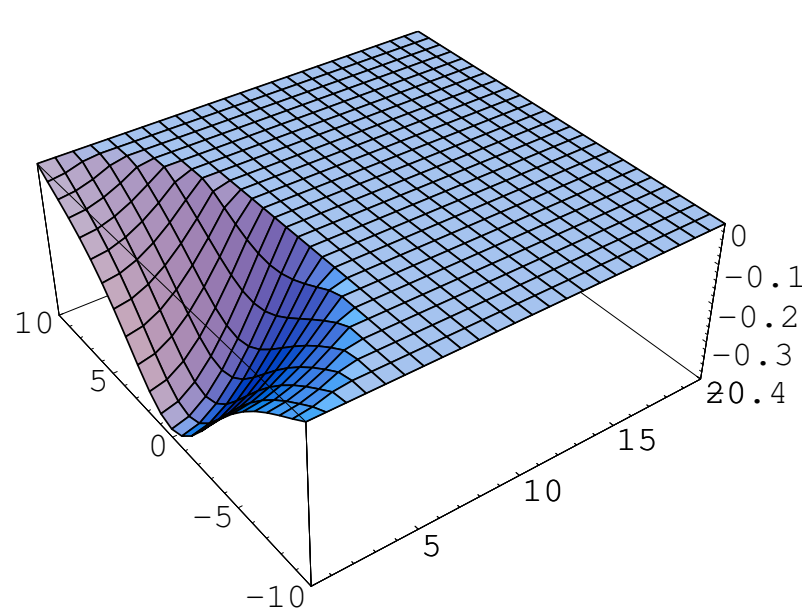

FIG. 7: Area of negative contributions to the density matrix $\rho_{E}(\mathbf{x}, \mathbf{x}+\mathbf{d})$ of a pointlike excitation for a shear of $20 \mathrm{~nm}$. The point and its image are situated at $(0,10)$ and at $(0,-10)$

(virtual) source.

In dipole approximation it is found that the density matrix of the inelastically scattered electrons is

$$
\bar{\rho}_{E}\left(\mathbf{x}, \mathbf{x}^{\prime}\right)=v(x) v\left(x^{\prime}\right) \mathbf{e}_{x} \cdot \mathbf{e}_{x^{\prime}}+w(x) w\left(x^{\prime}\right) .
$$

Here, $\mathbf{e}_{\mathbf{x}}$ is a unit vector in direction $\mathbf{x}$ in the exit plane. The two functions in eq.31 are[7]

$$
\begin{aligned}
& v=\frac{1}{2 \pi} \int_{0}^{\infty} \frac{q J_{1}(q x) f(Q)}{Q^{2}} d q, \\
& w=\frac{q_{E}}{2 \pi} \int_{0}^{\infty} \frac{J_{0}(q x) f(Q)}{Q^{2}} d q .
\end{aligned}
$$

Note that the scalar product $\mathbf{e}_{x} \cdot \mathbf{e}_{x^{\prime}}=\cos \phi$ is negative when the angle $\phi$ between directions $\mathbf{x}, \mathbf{x}^{\prime}$ is $>\pi / 2$.

Therefore the density matrix $\rho_{E}\left(\mathbf{x}, \mathbf{x}^{\prime}\right)$ has negative contributions, and we expect contrast inversion of the fringes (in other words, there is a phase shift of $\pi$ between some partial inelastically scattered waves). The results for a shear of $20 \mathrm{~nm}$ are shown in figs. 7 showing the area where the density matrix is negative (the positive parts wee set to zero in the figure so as to facilitate visualization) and in 8 showing the density matrix as a density plot (white is negative, black positive) and a simulation of the fringes. Contrast inversion can be observed in the semicircular area between the source and its image. note that both figures show only the right halfplane since everything is symmetric with respect to the line connecting source and image.

The minimum contrast is -0.37 ; it is found at the midpoint between the real and the virtual source. We propose therefore such an experiment with a small circle or sphere of a material with a well defined plasmon, such as $\mathrm{Al}$ or Si. Apart of the difficulty to prepare a specimen with almost pointlike excitation source surrounded 

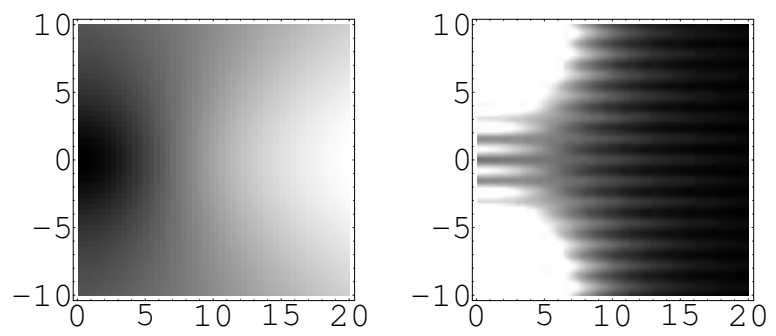

FIG. 8: Fringe contrast and fringes showing contrast inversion in the area in between a pointlike source of inelastic scattering and its image. The shear is $20 \mathrm{~nm}$ in vertical direction.

by vacuum the question is whether the intensity is high enough to detect the effect, and whether the strong intensity background from the point sources does not hide the minute fringe amplitudes. A simulation for an extended source is more tricky - we think that the contrast inversion will still be visible albeit with smaller amplitudes and in a smaller region. We expect visibility of the contrast inversion when the diameter of the excited region is well below the holographic shear.

\section{CONCLUSIONS}

We discussed several approaches to the correlation length in solid state plasmas. Results derived from the plasmon correlation function and its two-dimensional projection onto the plane of observation are in good agreement with earlier estimates.

It was further shown that the small correlation length in solid state plasmas does not contradict the coherence length of almost $30 \mathrm{~nm}$ found in inelastic interference experiments. The difference of nearly 2 orders of magnitude can be traced back to the long range Coulomb interaction between probe and target electrons. So it may happen that two partial waves emerging from the specimen a distance $d$ apart from each other have excited the same very localized - plasmon and thus are coherent over that large distance. Details of the electronic correlation in the scatterer are found in the first few $\mathrm{nm}$ of the mutual coherence function. Sophisticated experiments will be needed to reveal these details.

A surprising prediction of the density matrix approach is that inelastic holography experiments should show increased contrast outside the specimen, and contrast inversion of fringes in inelastic holograms of very small particles.

\section{Acknowledgments}

P. S. acknowledges the support of the Austrian Science Fund, project nr. P14038-PHY.

H. L. is grateful to Fondation Francqui, Brussels, for support of this research.
[1] N. Vast, L. Reining, V. Olevano, P. Schattschneider, and B. Jouffrey, PRL 88, 037601/1 (2002).

[2] R. DelSole, G. Adragna, V. Olevano, and L. Reining, Phys. Rev. B 67, 045207 (2003).

[3] H. Ness and A. Fisher, Chem. Phys. 281, 279 (2003).

[4] K. Liu, P. Avouris, and R. Martel, Phys. Rev. B 63, 161404(R) (2001).

[5] P. Schattschneider and B. Jouffrey, Plasmons and related excitations (1995), pp. 151-224.

[6] P. Schattschneider, Fundamentals of inelastic electron scattering (Springer, Wien, New York, 1986).

[7] P. Schattschneider, M. Nelhiebel, and B. Jouffrey, Phys. Rev. B 59, 10959 (1999).

[8] D. Pines, Elementary Excitations in Solids (W. A. Benjamin, New York, 1964).

[9] I. Egri, Z. Phys. B - cond. matter 53, 183 (1983).

[10] I. Egri, Phys. Reports 119, 6 pp. 363-402 (1985).

[11] R. Ferrell and J. Quinn, Phys.Rev. 108, No.3, 570 (1957).

[12] S. Cheng, Ultramicroscopy 21, 291 (1987).

[13] M. Scheinfein, A. Muray, and M. Isaacson, Ultramicroscopy 16, 233 (1985).

[14] A. Harscher, H. Lichte, and J. Mayer, Ultramicroscopy 69, 201 (1997).

[15] H. Lichte and B. Freitag, Ultramicroscopy 81, 177 (2000).
[16] H. Lichte, P. Potapov, D. van Dyck, and P. Schattschneider, in Microscopy and Microanalysis (Cambridge University Press, 2003), vol. 9, Suppl. 3, p. 084.

[17] S. L. Dudarev, L. M. Peng, and M. J. Whelan, Phys. Rev. B 48, 13408 (1993).

[18] H. Kohl and H. Rose, Adv. electronics and electron phys.65 pp. 173-227 (1985).

[19] P. Schattschneider, C. Hébert, and B. Jouffrey, Ultramicroscopy 86, 343 (2001).

[20] P. Schattschneider and B. Jouffrey, Ultramicroscopy 96, 453 (2003).

[21] L. Marton, Phys. Rev. 85, 1057 (1952).

[22] G. Pozzi, Optik 65, 77 (1983).

[23] f. Zhou, E. Plies, and G. Moellenstedt, Optik 98, 95 (1995).

[24] B. M. Mertens, M. Overwijk, , and P. Kruit, Ultramicroscopy $\mathbf{7 7}, 1$ (1999).

[25] G. Moellenstedt and H. Dueker, Z. Phys. 145, 377 (1956).

[26] H. Lichte, Phil Trans. R. Soc. Lond. A 360, 897 (2002).

[27] R. Speidel and D. Kurz, Optik 49, 173 (1977).

[28] D. van Dyck, H. Lichte, and J. Spence, Ultramicroscopy 81, 187 (2000).

[29] P. Hawkes, Coherence in electron optics (Academic Press, London, 1987), vol. 7, p. 101.

[30] H. Rose, Optik 45, 139 (1976).

[31] H. Müller, H. Rose, and P. Schorsch, J. Microscopy 190, 
73 (1998).

[32] P. Schattschneider and B. Jouffrey, Eur. Phys. J. B 37, 3 (2004).

[33] P. Potapov (2004).

[34] P. Schattschneider, M. Nelhiebel, H. Souchay, and B. Jouffrey, Micron 31, 333 (2000).

[35] This is more realistic than the Lorentzian argument given above since the angular distribution function in inelastic scattering all have a cutoff at relatively small angles

[36] For free space propagation, this is the Fresnel propaga- tor $\exp \left[-i \pi \frac{(\mathbf{r}-\mathbf{x})^{2}}{\lambda z}\right]$ which tends to the Fourier transform operator $\exp [-2 \pi i \mathbf{r x} / z]$ for $z \rightarrow \infty$

[37] In fact, the density matrix used in the following is the reduced one-electron density matrix of the probe electron, starting from the wave function of the entangled system.

[38] The description by $\cos (\mathrm{x})$ is problematic now since the $\Delta \phi$ can be different for the different incoherent waves.

[39] In diffraction, a similar effect was already observed[34]. 